\section{P1-319 A MULTILEVEL APPROACH FOR STUDYING THE ASSOCIATION BETWEEN NEIGHBOURHOOD CHARACTERISTICS AND SELF-RATED HEALTH IN THE BRAZILIAN PRÓ-SAÚDE STUDY}

doi:10.1136/jech.2011.142976f.11

${ }^{1} \mathrm{~S}$ Santos, ${ }^{* 1} \mathrm{D}$ Chor, ${ }^{1,2} \mathrm{G}$ Werneck, ${ }^{2} \mathrm{C}$ Lopes, ${ }^{2}$ E Faerstein. ${ }^{1}$ National School of Public Health, Oswaldo Cruz Foundation, Rio de Janeiro, Brazil; ${ }^{2}$ Social Medicine Institute, Rio de Janeiro Satate University, Rio de Janeiro, Brazil

Introduction Few studies examine the influence of neighbourhood characteristics on self-rated health (SRH), particularly in middleincome countries such as Brazil. We examine the association between neighbourhood of residence indicators of socioeconomic position and individual self-rated health in the Pró-Saúde cohort study, adjusting for group and individual characteristics.

Methods A multilevel approach using hierarchical modelling was applied to analyse the relationship between neighbourhood indicators of socioeconomic position and self-rated health in 3054 University employees living in Rio de Janeiro city who participate in the Pró-Saúde study. Neighbourhoods $(\mathrm{n}=621)$ were created using the SKATER algorithm, a tool for area regionalisation to group small areas (TerraView software). Census tracts were grouped based on four census tract indicators and a minimum population of 5000 living in the delimited neighbourhood.

Results Adjusting for individual factors, such as individual income per capita, educational level, age, sex, ethnicity, health related behaviours and the presence of chronic diseases, low neighbourhood income level and a higher number of inhabitants per residence were significantly associated with poor SRH. Subjects living in neighbourhoods with medium income level were 34\% more likely to rated their health as poor. Those living in areas with a higher density of persons per household were $50 \%$ more likely to report poor SRH. Conclusion Following adjustment for individuals factors neighbourhood context influenced SRH in this study; poor neighbourhood socioeconomic conditions are associated with poor SRH.

\section{P1-320 EFFECTS OF A 6 MONTH COMMUNITY-BASED LIFESTYLE MODIFICATION PROGRAM ON METABOLIC PARAMETERS AND DIETARY PATTERNS IN THE RURAL JAPANESE OVER 40 YEARS OLD}

doi:10.1136/jech.2011.142976f.12

${ }^{1} \mathrm{~F}$ Sata, ${ }^{*}{ }^{1} \mathrm{H}$ Imai, ${ }^{1} \mathrm{H}$ Nakao, ${ }^{2} \mathrm{Y}$ Fukuda. ${ }^{1}$ National Institute of Public Health, Wako, Saitama, Japan; ${ }^{2}$ Yamaguchi University School of Medicine, Ube, Yamaguchi, Japan

Introduction In Japan, a new screening and intervention program specifically targeting the metabolic syndrome has been conducted since April 2008. The aim of the study was to evaluate the effects of a 6 month lifestyle modification program on metabolic parameters and dietary patterns in the rural Japanese over 40 years old.

Methods 159 rural inhabitants over 40 years old with metabolic syndrome risk factors, were randomly allocated into intensive intervention $(n=79)$ and standard care control $(n=80)$ groups. The subjects in the intervention group participated in a 6-month lifestyle modification program consisting of health education, exercise, diet, or counselling every month with self-monitoring body weight, blood pressure and fasting blood glucose (FBG) everyday, whereas those in the control group participated in a standard care program consisting of health education without self-monitoring. Food frequency questionnaires and health examination were conducted before and after the program.

Results The participants in the intervention group showed significant reductions in daily intake of carbohydrates $(p<0.05)$ and fruits $(p<0.01)$, body weight $(p<0.001)$, waist circumference $(p<0.001)$, systolic and diastolic blood pressure (both $p<0.001)$, FBG $(p<0.05)$, haemoglobin A1c $(p<0.05)$, triglyceride $(p<0.001)$ and a significant gain in HDL-cholesterol $(p<0.001)$, whereas those in the control group showed significant reductions only in body weight $(p<0.001)$, waist circumference $(p<0.001)$, systolic and diastolic blood pressure $(p<0.05$ and $p<0.001$, respectively). The men in the intervention group showed significant reductions in daily intake of alcohol $(\mathrm{p}<0.05)$.

Conclusion The intensive intervention program was more effective in reducing multiple metabolic parameters and dietary consumption than the standard care one.

\section{P1-321 STATISTICAL MODELLING TECHNIQUE IN FORECASTING OF PALLIATIVE ONCOTHERAPY LOAD IN HOSPITALS}

doi:10.1136/jech.2011.142976f.13

${ }^{1} B$ Sathian, * 2J Sreedharan, ${ }^{1} \mathrm{~K}$ Sharan, ${ }^{1} \mathrm{~S}$ Baboo, ${ }^{1} \mathrm{~N}$ Chandrasekharan, ${ }^{3} \mathrm{E}$ Rajesh. ${ }^{1}$ Manipal College of Medical Sciences, Pokhara, Nepal; ${ }^{2}$ Gulf Medical University, Ajman, United Arab Emirates; ${ }^{3} M G$ University, Kerala, India

Background An increase in cancer incidence in developing countries is expected in future and the number of cancer patients requiring palliative treatment will also rise in Nepal. Estimation of trends is very essential for the planning of future requirements in any healthcare programme.

Method This retrospective study analysed the records from the Radiotherapy Department at Manipal Teaching Hospital, Pokhara, Nepal to calculate the number of cancer patients who received palliative treatment with radiotherapy between September 2000 and December 2008. Statistical modelling techniques were applied for forecasting of future trends.

Results Of 1001 cancer cases, 363 patients received radiotherapy with palliative intent during the study period. Excluding the constant term from the equation, the Logarithmic model was the best fitted, with $\mathrm{R} 2=0.727, \mathrm{p}=0.002$ for the forecasting of cancer patients receiving palliative treatment. Using this model, the number of cancer cases receiving palliative radiotherapy at the hospital in the year 2015 was estimated to be 68 . Thus, the number of patients requiring radiotherapy with palliative intent shows a pattern of increasing trend over the coming years.

Conclusion The data analysed in the present study indicates an increasing future trend of patients requiring palliative therapy at the centre. Therefore, in the future, there could be a discrepancy between the requirement and capacity of care to these patients. Government and healthcare agencies of Nepal must ready themselves to promote better strategies for adequate provision to the cancer patients receiving palliative treatment in the coming years.

\section{P1-322 STATISTICAL MODELLING IN THE PREDICTION OF KALA- AZAR IN NEPAL}

doi:10.1136/jech.2011.142976f.14

${ }^{1} B$ Sathian, ${ }^{*} \mathrm{~J}$ Sreedharan, ${ }^{1} \mathrm{~N}$ Chandrasekharan, ${ }^{1} \mathrm{~S}$ Devkota, ${ }^{3}$ E Rajesh, ${ }^{1} \mathrm{~A}$ Mittal. ${ }^{1}$ Manipal College of Medical Sciences, Pokhara, Nepal; ${ }^{2}$ Gulf Medical University, Ajman, United Arab Emirates, ${ }^{3} M G$ University, Kerala, India

Background Kala-azar (Visceral Leishmaniasis) is endemic in 12 Terai districts of Nepal. More than 5.6 million people living in these districts are believed to be at risk of developing Kala-azar. The objective of this study was to predict future trends in Kala-azar using available data.

Methods We carried out a retrospective study using data collected from the Health Ministry records of Nepal, between 2003 and 2007. Descriptive statistics and statistical modelling were used for analyses. 